\title{
Critical involvement of the $\alpha(1,2)$-fucosyltransferase in multidrug resistance of human chronic myeloid leukemia
}

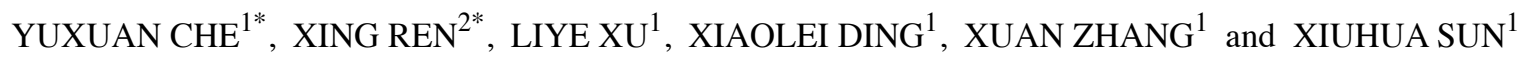 \\ ${ }^{1}$ Department of Medical Oncology, The Second Affiliated Hospital of Dalian Medical University, Dalian, \\ Liaoning 116027; ${ }^{2}$ College of Stomatology, Dalian Medical University, Dalian, Liaoning 116027, P.R. China
}

Received December 6, 2015; Accepted January 11, 2016

DOI: $10.3892 /$ or.2016.4673

\begin{abstract}
The fucosyltransferases are key enzymes in cell surface antigen synthesis during multidrug resistance (MDR) development. The aim of the present study was to analyze the alteration of $\alpha(1,2)$-fucosyltransferase involved in MDR development in human chronic myeloid leukemia (CML). FUT1 was overexpressed in three CML/MDR cell lines and peripheral blood mononuclear cells (PBMC) of CML patients. However, no significant changes of FUT2 were observed. The altered levels of FUT1 had a significant impact on the phenotypic variation of MDR of K562 and K562/ADR cells, the activity of EGFR/MAPK pathway and P-glycoprotein (P-gp) expression. Blocking the EGFR/MAPK pathway by its specific inhibitor PD153035 or EGFR small interfering RNA (siRNA) resulted in the reduced MDR of K562/ADR cells. This study indicated that $\alpha(1,2)$-fucosyltransferase involved in the development of MDR of CML cells probably through FUT1 regulated the activity of EGFR/MAPK signaling pathway and the expression of P-gp.
\end{abstract}

\section{Introduction}

Chronic myeloid leukemia (CML) is a clonal hematopoietic stem cell disorder characterized by the increased growth of

Correspondence to: Dr Xiuhua Sun, Department of Medical Oncology, The Second Affiliated Hospital of Dalian Medical University, 467 Zhongshan Road, Dalian, Liaoning 116027, P.R. China

E-mail: sunxh1970@163.com

${ }^{*}$ Contributed equally

Abbreviations: FUT, fucosyltransferase gene; MDR, multidrug resistance; CML, chronic myeloid leukemia; PBMC, peripheral blood mononuclear cells; siRNA, small interfering RNA; shRNA, short hairpin RNA; P-gp, P-glycoprotein; PBS; phosphate-buffered saline; PBST, containing 0.1\% Tween-20; ADR, adriamycin; DMSO, dimethyl sulfoxide; MTT, methylthiazolyl tetrazolium; IHC, immunohistochemistry

Key words: FUT1, multidrug resistance, chronic myeloid leukemia, EGFR/MAPK signaling, P-glycoprotein predominantly myeloid cells in the bone marrow and the accumulation of these cells in the blood (1). Multidrug resistance (MDR) is a major challenge to the successful treatment of CML. The mechanisms which generate the MDR phenotype of CML cells are complex, including increase of drug excretion, antiapoptosis, activity changes in drug metabolizing enzymes, enhancement of DNA repair following damage, changes in signaling pathway. Research has recently been devoted to understanding the interaction of glycan alterations and resistance to chemotherapy of cancer cells.

Glycans attached to proteins are ubiquitous in biological systems, and many proteins in eukaryotes are glycosylated (2). Most of these are N-linked and/or O-linked glycan chains that are synthesized by various glycosyltransferases (3). The fucosyltransferase gene family encodes enzymes that transfer fucose from $\alpha(1,2), \alpha(1,3 / 4)$ and $\alpha(1,6)$ linkages to various glycans. Fucosyltransferase 1 (FUT1) and FUT2 are $\alpha(1,2)$ fucosyltransferases responsible for synthesis of the $\mathrm{H}$ blood group antigen $(4,5)$. The expression of cancer-associated carbohydrate antigens is modified by abnormal control by glycosyltransferases. In human colon adenocarcinoma, expression of the fucosyltransferase gene FUT1 has been found to correlate with malignant progression (6). Downregulation of microRNA-15b by hepatitis B virus X enhanced hepatocellular carcinoma proliferation via fucosyltransferase 2-induced Globo H expression (7). In addition, forced FUT1 and FUT2 expression in human ovarian carcinoma-derived RMG-I cells promoted cell proliferation and resistance against anticancer drugs, such as 5-fluorouracil and carboplatin $(8,9)$. However, little information is available on the reversal effects of FUT1 and FUT2 glycosyltransferases and corresponding glycogenes on multi-drug resistance in human CML cells.

Epidermal growth factor receptor (EGFR) has attracted much attention for its potency in regulating cell activation. Binding of ligands such as EGF, the tyrosine specific protein kinase intrinsic to EGFR, results in activation, and is followed by transactivation of mitogen activated protein kinase (MAPK) and other downstream signal pathways (10). In addition, several reports highlight that aberrant activation of EGFR/MAPK pathway contributes to the drug resistance of different types of human cancer cells. Non-small cell lung cancer cells with acquired resistance to cetuximab manifested strong activation of EGFR and MAPK (11). Blockade of the EGF receptor (EGFR)/MAPK pathway caused more marked inhibition of 
growth of tamoxifen-resistant MCF-7 breast cancer cells (12). The uncoupling of EGFR with mitogenic pathways caused resistance to EGFR inhibition in bladder cancer (13). A potential association of FUT1 with EGFR signaling pathway has been explored as well. Knocking down FUT1 expression inhibited human epidermoid carcinoma A431 cell proliferation through decreasing the EGFR signaling pathway (19).

In the present study, we investigated the mRNA expression levels of $\alpha(1,2)$-fucosyltransferase genes in three pairs of parental and chemoresistant CML cell lines and in BMMC isolated from the diagnostic CML patients. We further determined the functional role of FUT1 in CML MDR, as well as the possible mechanisms via EGFR/MAPK pathway.

\section{Materials and methods}

Parental CML cell culture. Three CML cell lines, K562, KCL22 and KU812, were purchased from the Nanjing KeyGen Biotech (Co., Ltd., Nanjing, China). All cell lines were cultured in RPMI-1640 medium (Gibco, Grand Island, NY, USA) supplemented with $1 \%$ penicillin-streptomycin (Gibco) and $10 \%$ heat inactivated fetal bovine serum (FBS; Gibco). Cells were kept a humidified incubator at $37^{\circ} \mathrm{C}$ with $5 \% \mathrm{CO}_{2}$. Adriamycin (Sigma) was added to parental cell cultures in stepwise increasing concentrations from 0.1 to $5 \mu \mathrm{g} / \mathrm{ml}$ for 4 months to develop an adriamycin-resistant (ADR) clone. Once ADR-resistant clones became resistant, the complete medium of the resistant cell clones were supplemented with $1.0 \mathrm{mg} / \mathrm{l}$ adriamycin. Over $90 \%$ of ADR cells were susceptible to subsequent treatments if they were maintained in complete medium without adriamycin for one week.

Samples from leukemia patients and primary CML peripheral blood mononuclear cells (PBMCs). Thirty-nine previously untreated CML patients and 9 healthy donors were included in this study. The diagnosis of CML was based on cytomorphology, cytochemistry, multiparameter flow cytometry, immunology, molecular genetics and cytogenetics. There were 21 males and 18 females with age ranging from 19 to 71 years (median age, 43 years). P-gp (+) was observed in 22 of 39 CML patients. All the participants recruited from Jan 2012 to Dec 2014 at the Second Affiliated Hospital of Dalian Medical University (Dalian, China) provided written informed consent. The investigation project and the informed consent were examined and certified by the Ethics Committee of the Second Affiliated Hospital of Dalian Medical University.

PBMCs from CML patients were separated by FicollHypaque density gradient centrifugation and were further cultured in plastic dishes to remove adherent cells at $37^{\circ} \mathrm{C}$ for $24 \mathrm{~h}$. Fresh separated non-adherent cells were maintained in modified Dulbecco's medium containing 10\% FBS, $10 \mathrm{mM}$ $\beta$-mercaptoethanol, $2 \mathrm{mM}$ L-glutamine, $50 \mathrm{ng} / \mathrm{ml}$ human stem cell factor, $10 \mathrm{ng} / \mathrm{ml}$ human interleukin-3 and $10 \mathrm{ng} / \mathrm{ml}$ human interleukin-6. Cells were then harvested and real-time PCR analysis was completed.

Real-time PCR analysis. Total RNA was isolated with TRIzol reagents (Gibco-BRL, Rockville, MD, USA), and cDNA was synthesized using QuantiTect reverse transcription kit (Qiagen, Valencia, CA, USA) according to the manufacturer's instruc- tion. Quantitative PCR with QuantiTect SYBR-Green (Qiagen) was performed for each transcript. The following primers were used: 5'-AAAGCGGACTGTGGATCT-3' and 5'-GGA CACA G GATCGACAGG-3' for FUT1; 5'-CTGCCCAACCA CTCTGTC-3' and 5'-CCGTAAAGACAAAGAGGATG-3' for FUT2; 5'-CTCCTCCACCTTTGACGCTG-3' and 5'-TCCTC TTGTGCTCTTGCTGG-3' for GAPDH. Finally expression of the transgenes was determined quantitatively by the relative $\mathrm{Ct}$ method. The relative gene expression was normalized to that of respective GAPDH and calculated as $2-\left(\mathrm{Ct}_{\text {Target gene }}-\mathrm{Ct}_{\mathrm{GAPDH}}\right)$.

Western blot analysis. Whole cell lysates were subjected to sodium dodecyl sulfate-polyacrylamide gel electrophoresis and transferred to polyvinyledene fluoride (PVDF) membranes (Bio-Rad Laboratories, Hercules, CA, USA). After blocking with 5\% skimmed milk in PBS containing $0.1 \%$ Tween-20 (PBST), the membrane was incubated with antibody $(1 / 200$ diluted; Santa Cruz Biotechnology) and then with peroxidaseconjugated anti-rabbit IgG (1/10,000 diluted; GE Healthcare UK, Little Chalfont, UK). A GAPDH antibody (1/200 diluted; Santa Cruz Biotechnology) was used as a control. The protein bands on the membrane were visualized using the Western Lightning chemiluminescence reagent (PerkinElmer, Waltham, MA, USA). The bands were analyzed with LabWorks $^{\mathrm{TM}}$ ver4.6; UVP, BioImaging systems).

shRNA-mediated FUT1 gene silencing. K562/ADR cells were incubated in appropriate antibiotic-free medium with $10 \%$ fetal bovine serum, and were transferred to a 6-well tissue culture and incubated at $37^{\circ} \mathrm{C}$, in a $\mathrm{CO}_{2}$ incubator to obtain $60-80 \%$ confluence. The cell cultures were transfected with FUT1-specific shRNA, and scrambled shRNA used as the negative control. FUT1 shRNA was mixed with Lipofectamine 2000 (Invitrogen, Carlsbad, CA, USA). Transfected cells were cultured and incubated at $37^{\circ} \mathrm{C}$ for $6 \mathrm{~h}$, followed by incubation with complete medium for additional $24 \mathrm{~h}$. Then, the cells were harvested for further study. The cell transfection efficiency was $73 \%$ by fluorescent microscopy and the cell viability was $85 \%$ by trypan blue dye exclusion assay.

Overexpression of FUT1. The human FUT1 coding sequences obtained from Takara Co. (Dalian, China) were inserted into the pEGFP-N2 vector (Invitrogen) at the sites of EcoRI and XhoI. K562 cells were transfected with $5 \mu \mathrm{g}$ of target gene expression vector or empty vector (EV) in 100-mm dishes using PolyFect transfection reagent (Qiagen) according to the manufacturer's instruction. After 4 weeks of screening, the cell lines stably expressing FUT1 (K562/FUT1), and cells with empty vector (HL60/mock) were established. The cell transfection efficiency was $75 \%$ and the survival rate was $87 \%$.

In vitro drug sensitivity assay. Cell drug sensitivity was measured using an MTT assay. The K562/ADR, K562/ADR-control shRNA, K562/ADR-FUT1 shRNA, K562, K562/mock, K562/FUT1 and K562/ADR with DMSO, PD153035, control siRNA or EGFR siRNA treatment cells $\left(1 \times 10^{4}\right)$ were grown in 96-well plates and incubated with different anticancer drugs adriamycin, vincristine and paclitaxel (Sigma, St. Louis, MO, USA) for 48 h, respectively. Then, 
A

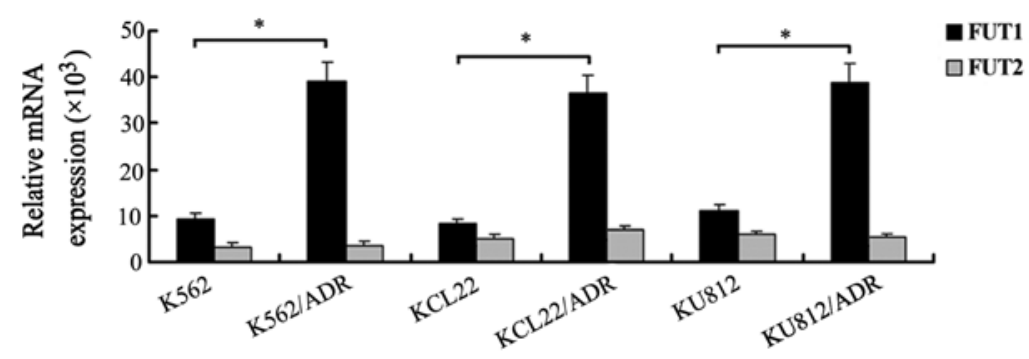

B
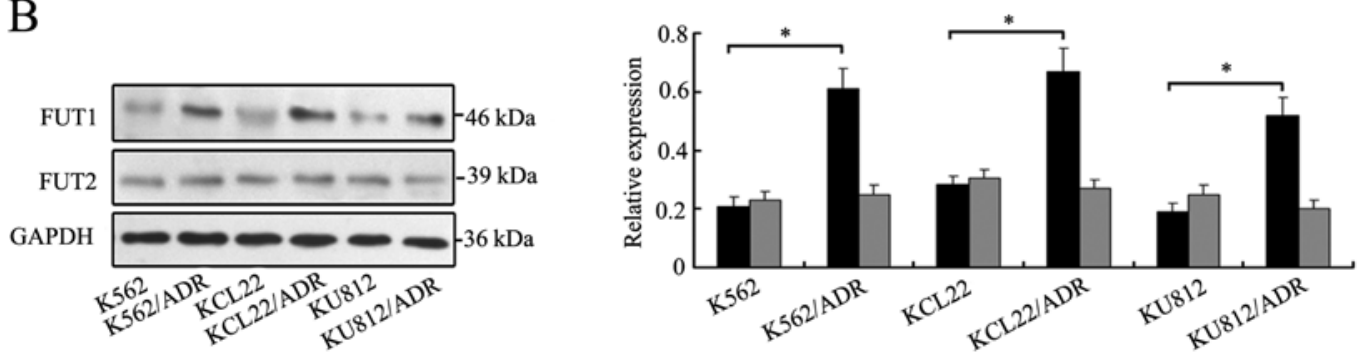

Figure 1. Differential expression of FUT1, FUT2 in three pairs of parental and chemoresistant human CML cell lines and CML patients. (A) The mRNA levels of FUT1 and FUT2 analyzed by real-time RT-PCR. The relative amount of gene mRNA level was normalized to GAPDH level. Three MDR cells expressed higher levels of FUT1 mRNA than their parental cell types ( $>3$-fold; $\left.{ }^{2} \mathrm{P}<0.05\right)$. No significant change of FUT2 was observed. (B) Western blot analysis of FUT1 and FUT2 at protein levels. GAPDH served as a control. Three MDR cells expressed higher levels of FUT1 protein $\left({ }^{*} \mathrm{P}<0.05\right)$. Data are the means \pm SD of triplicate determinants.

the cells were treated with $100 \mu 1 \mathrm{MTT}$ ( $5 \mathrm{mg} / \mathrm{ml}$; Sigma). After 4-h incubation at $37^{\circ} \mathrm{C}$ in $5 \% \mathrm{CO}_{2}, 100 \mu \mathrm{l}$ dimethyl sulfoxide (DMSO; Gibco) was added to dissolve formazan crystals that formed and the absorbance was measured at $490 \mathrm{~nm}$ using microplate reader (BioTek Instruments, Inc., Winooski, VT, USA). The drug resistance was estimated by comparing the $\mathrm{IC}_{50}$ values (the drug concentration that inhibits cell growth by $50 \%$ ) from growth inhibition curves.

In vivo chemosensitivity assay. Approval for animal studies was obtained from the Dalian Medical University Institutional Animal Care and Use Committees. Five-week-old male athymic nude mice were obtained from the Animal Facility of Dalian Medical University, and were fed with sterilized food and water. Approximately, $1 \times 10^{7}$ cells were injected subcutaneously into the right flank of each nude mouse, respectively. One week after tumor cell injection, tumor-bearing mice were randomly divided into control and treatment groups $(n=6$ animals per group). The treatment groups received $7 \mathrm{mg} / \mathrm{kg}$ adriamycin i.p. three times a week for 3 weeks, and the control groups received physiological saline alone. The mice were sacrificed and their tumors were isolated, weighed and photographed. The tumor volume was calculated by the following formula: Tumor volume $=1 / 2\left(\right.$ length $\mathrm{x}$ width $\left.{ }^{2}\right)$.

Immunohistochemical (IHC) staining analysis. Visible tumors were removed from the mice and immunohistochemistry was performed on paraffin-embedded tissue sections using the fully automated Dako immunohistochemistry staining system (Autostainer Link 48; Dako, Glostrup, Denmark). The slides were dried, deparaffinized and rehydrated. After deparaffinization and blocking of endogenous peroxidase, the slides were labeled with antibodies (Abcam, Cambridge, UK) at a dilution of 1:200 at $4^{\circ} \mathrm{C}$ overnight. The secondary streptavidin-HRP-conjugated antibody staining (Santa Cruz Biotechnology) was performed at room temperature for
$60 \mathrm{~min}$. Finally, the sections were counterstained with hematoxylin and coverslipped.

Inhibition of the EGFR/MAPK signaling. PD153035 (Sigma) or EGFR siRNA was applied to suppress the activity of the EGFR/MAPK signaling in K562/ADR cells. Briefly, the leukemia cells $\left(1 \times 10^{4}\right.$ cells/well) were incubated in DMSO supplemented with the EGFR inhibitor PD153035 $(10 \mu \mathrm{M})$. EGFR control siRNA and EGFR siRNA cells were collected after $24 \mathrm{~h}$.

Flow cytometric analysis. Expression of $\alpha-(1,2)$ fucosylation at the cell surface was analyzed by flow cytometry using FITC-UEA-1 lectin (Sigma). Expression of P-gp at the cell surface was incubated with anti-P-gp antibody. After repeated centrifugation at $1,000 \mathrm{r} / \mathrm{min}$, labeled cells were resuspended in $0.2 \mathrm{ml} \mathrm{PBS}$ and were analyzed with FACSCalibur (BD Biosciences, San Jose, CA, USA). For mean fluorescence intensity, each value of the geometric mean was calculated by CellQuest software.

Statistical analysis. The data from the triple tests of each group were expressed as the mean \pm SD and analyzed by the SPSS 16.0 statistical software to evaluate the statistical difference. The Student's t-tests were used to compare the significance of differences among the examined groups. A statistically significant difference was considered at $\mathrm{P}<0.05$.

\section{Results}

Differential expression of FUT1, FUT2 in three pairs of parental and chemoresistant human CML cell lines and CML patients. To increase our understanding of the regulation of $\alpha(1,2)$-fucosylation, we determined the mRNA levels of FUT1 and FUT2 in drug sensitive and MDR cells. As shown in Fig. 1A, three MDR cell lines showed elevated levels of FUT1 
A
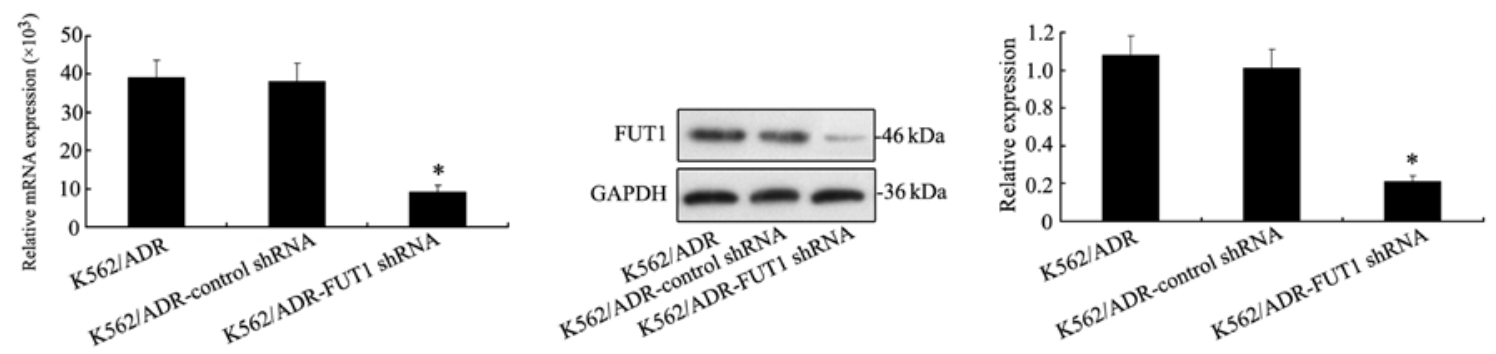

B
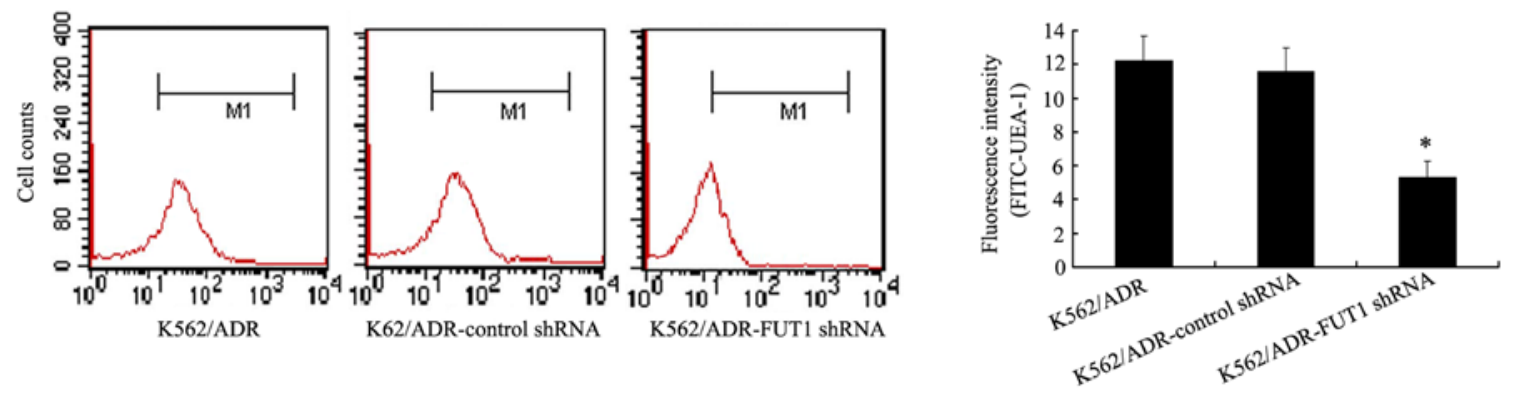

C

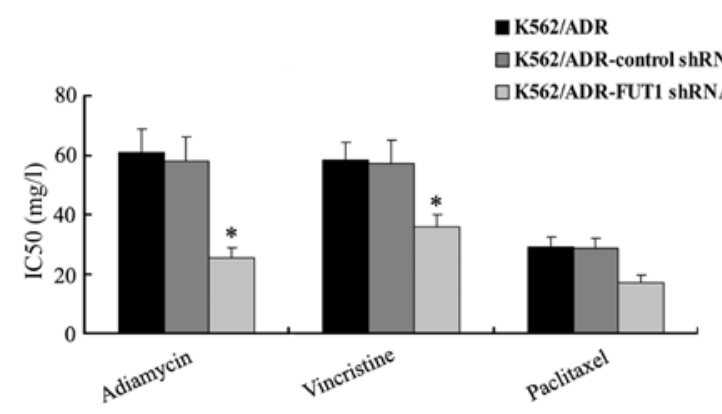

$\mathrm{E}$

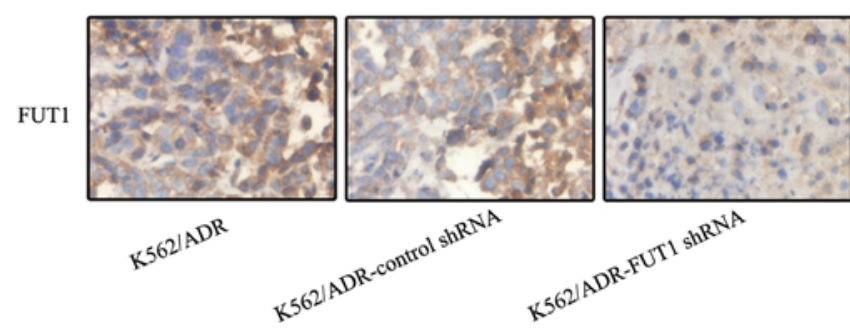

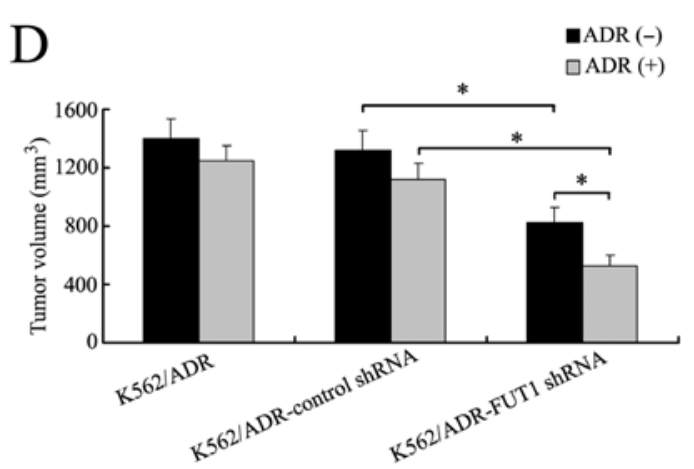

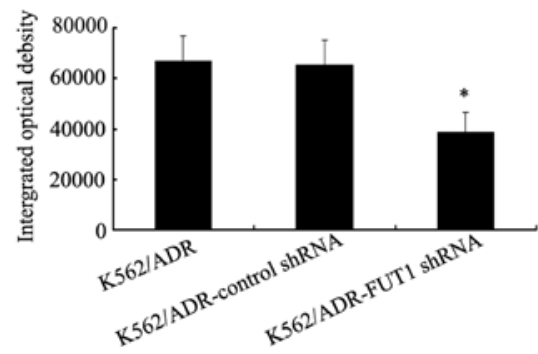

Figure 2. Downregulation of FUT1 gene enhances chemosensitivity of cells in vitro and in vivo. (A) Silencing of FUT1 in K562/ADR cells was analyzed by RNAi approach. After shRNA transfection, distinct reduction of FUT1 was observed at mRNA and protein levels by real-time RT-PCR and western blot analysis. (B) FITC-UEA-1 binding profile of FUT1 shRNA cells using flow cytometry. Histograms of fluorescence intensities of cells with specific carbohydrate expression as determined. (C) Cell chemosensitivity was assessed by cytotoxicity assays. The reported values were the IC ${ }_{50}$ (mean \pm SD) of three independent experiments. $\mathrm{IC}_{50}$ represents the drug concentration producing $50 \%$ decrease of cell growth. ${ }^{*} \mathrm{P}<0.05$ vs. K562/ADR-control shRNA cells. (D) A decrease of mean tumor volume in the mouse group with K562/ADR-FUT1 tumor was observed, as compared with that in K562/ADR group and K562/ADRcontrol shRNA group ( $\left.{ }^{*} \mathrm{P}<0.05\right)$. Downregulation of FUT1 was also shown by IHC staining (E) in xenograft tumors derived from K562/ADR-FUT1 shRNA cells (magnification, $\mathrm{x} 400)$. The data are means \pm SD of 3 independent assays $\left({ }^{*} \mathrm{P}<0.05\right)$.

expression compared with the three drug-sensitive parental cell lines. By contrast, no significant change of FUT2 was observed (Fig. 1A). Western blot analysis further confirmed the protein expression levels of FUT1 and FUT2 in drug sensitive and MDR cells (Fig. 1B).

Expression of MDR-related marker, FUT1, FUT2 present in peripheral blood mononuclear cells of CML patients is summarized in Table I. The frequency of P-gp positivity was $56.4 \%$ (22 of 39 ) in the CML patients. The mRNA expression levels of FUT1 and FUT2 were measured in the PBMC of CML without MDR and CML/MDR by real-time PCR.
Table I. Expressional profiles of FUT1 and FUT2 in CML and CML/MDR patients.

\begin{tabular}{lrrr}
\hline & \multicolumn{2}{c}{ Relative mRNA expression $\left(\times 10^{3}\right)$} & \\
\cline { 2 - 3 } Gene & \multicolumn{1}{c}{ CML } & CML/MDR & P-value \\
\hline FUT1 & $15.466 \pm 1.094$ & $32.101 \pm 7.382$ & $0.004^{\mathrm{a}}$ \\
FUT2 & $1.088 \pm 0.245$ & $1.236 \pm 0.519$ & 0.699 \\
\hline
\end{tabular}

${ }^{a} \mathrm{P}<0.05$ vs CML patients. 
A
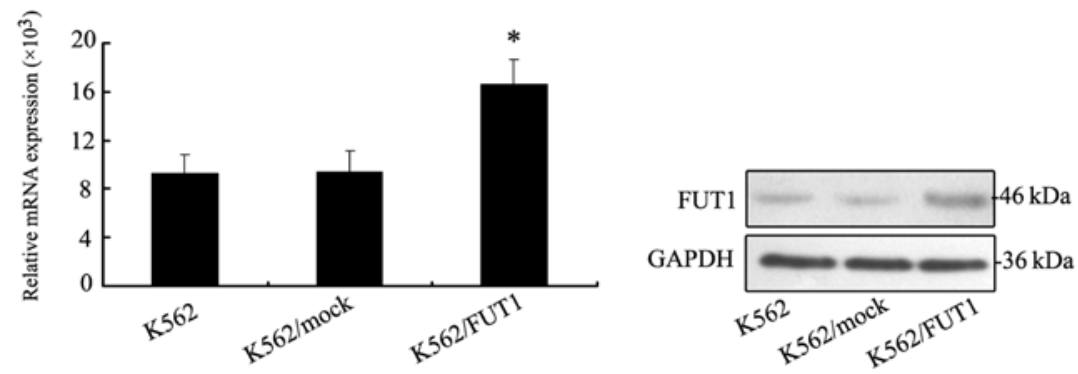

$\mathrm{B}$
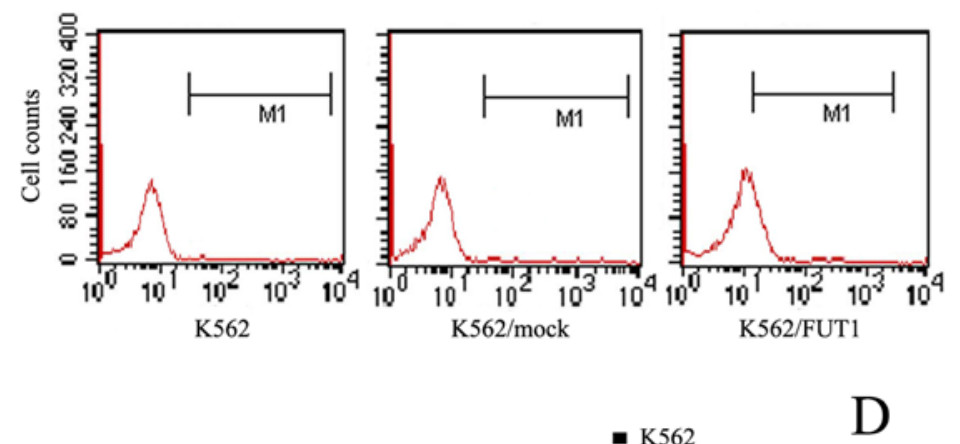

$\mathrm{C}$

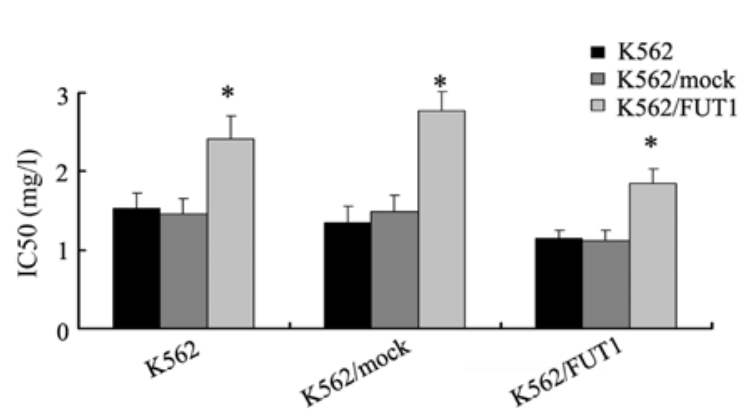

$\mathrm{E}$
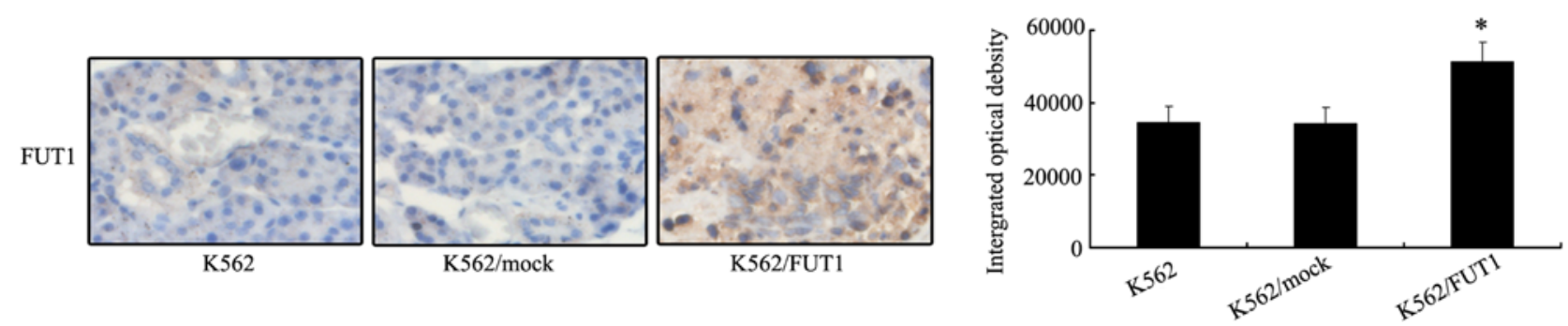

Figure 3. Overexpression of FUT1 gene enhances the chemoresistance of K562 cells both in vitro and in vivo. (A) After full-length sequences transfection, FUT1 mRNA and protein were increased notably in K562 cells by real-time PCR and western blot analysis. (B) FITC-UEA-1 binding profile of K562/FUT1 cells using flow cytometry. Histograms of fluorescence intensities of cells with specific carbohydrate expression as determined. (C) Cell chemosensitivity was assessed by cytotoxicity assays. The reported values were the $\mathrm{IC}_{50}$ (mean $\pm \mathrm{SD}$ ) of three independent experiments. $\mathrm{IC}_{50}$ represents the drug concentration producing $50 \%$ decrease of cell growth. ${ }^{*} \mathrm{P}<0.05$ vs. K562/mock cells. (D) An increase of mean tumor in the mouse group with K562/FUT1 tumor was observed, as compared with that in K562 group and K562/mock group ("P<0.05). (E) Upregulation of FUT1 by IHC staining in xenograft tumors derived from K562/ FUT1 cells (magnification, $\mathrm{x} 400$ ). The data are means \pm SD of 3 independent assays $\left({ }^{*} \mathrm{P}<0.05\right)$.

The group of CML/MDR showed significantly higher level of FUT1 ( $\mathrm{P}=0.004)$ mRNA expression than one of the chemosensitive groups. Expression of FUT2 showed no difference in expression levels between the two groups. These observations indicated that the differential expression of FUT1 might contribute to MDR of CML.

Downregulation of FUT1 gene enhances chemosensitivity of K562/ADR cells in vitro and in vivo. Due to the significant increase of FUT1 mRNA and protein expression in K562/ADR cells, we silenced FUT1 with shRNA to elucidate the direct implication of FUT1 in the chemosensitivity of K562/ADR cells. As shown in Fig. 2A, the expression level of FUT1 was significantly decreased in FUT1 shRNA transfectants compared to those in control. Furthermore, flow cytometric analysis was performed to study $\alpha-(1,2)$ fucosylation level on cell surface by using FITC-UEA-1. A typical image is shown in Fig. 2B. Results from three independent experiments demonstrated that the mean fluorescence intensity was reduced in K562/ADR-FUT1 shRNA cells. Significant differences were seen across cell lines (K562/ADR-FUT1 shRNA cells to control shRNA cells, $\mathrm{P}<0.05)$. These results clearly showed that FUT1 was responsible for the overcoming of tumor cell MDR resistance via regulating fucosylation profile in terms of $\alpha-1,2$ branched structures in CML cells. 

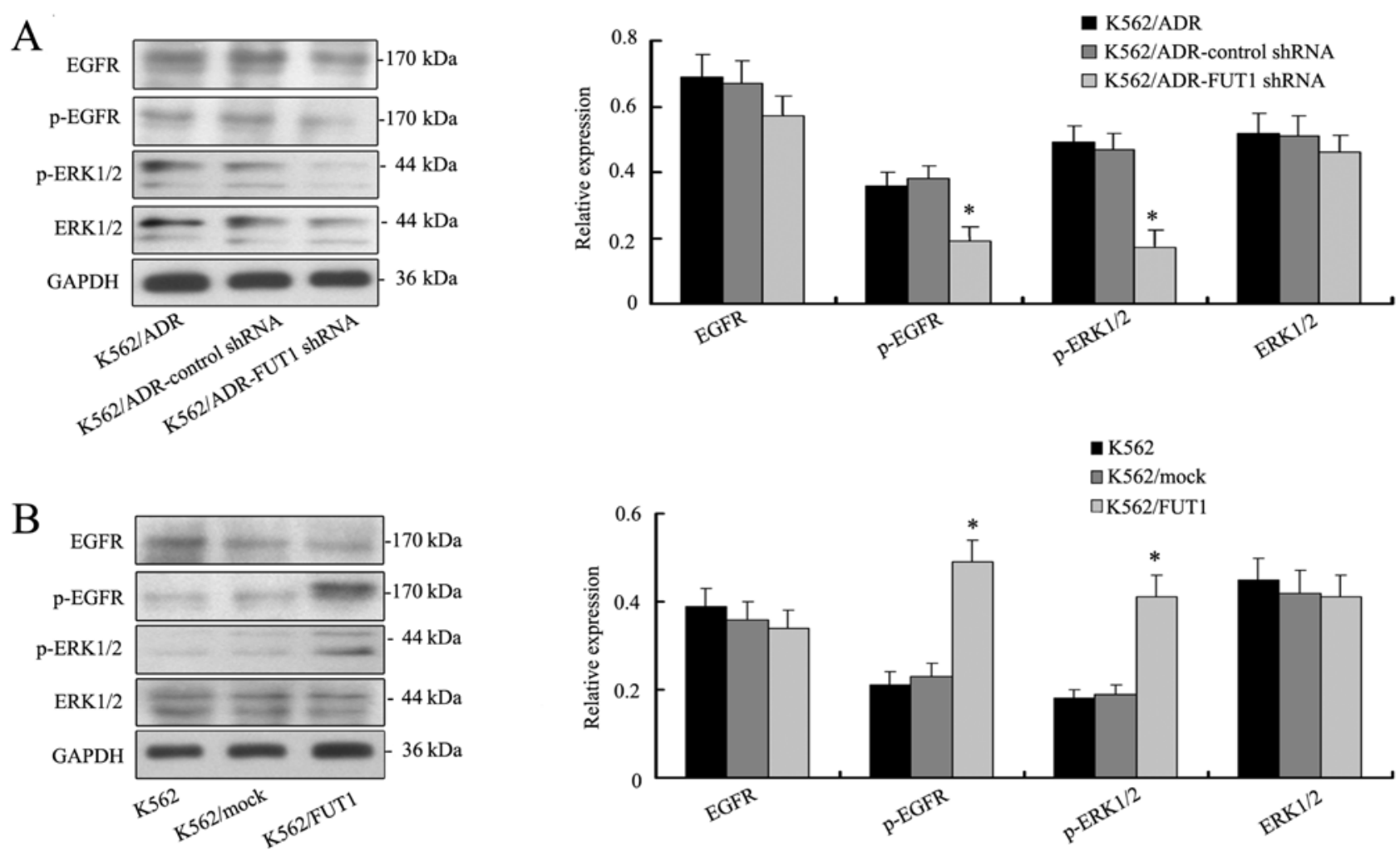

$\mathrm{C}$
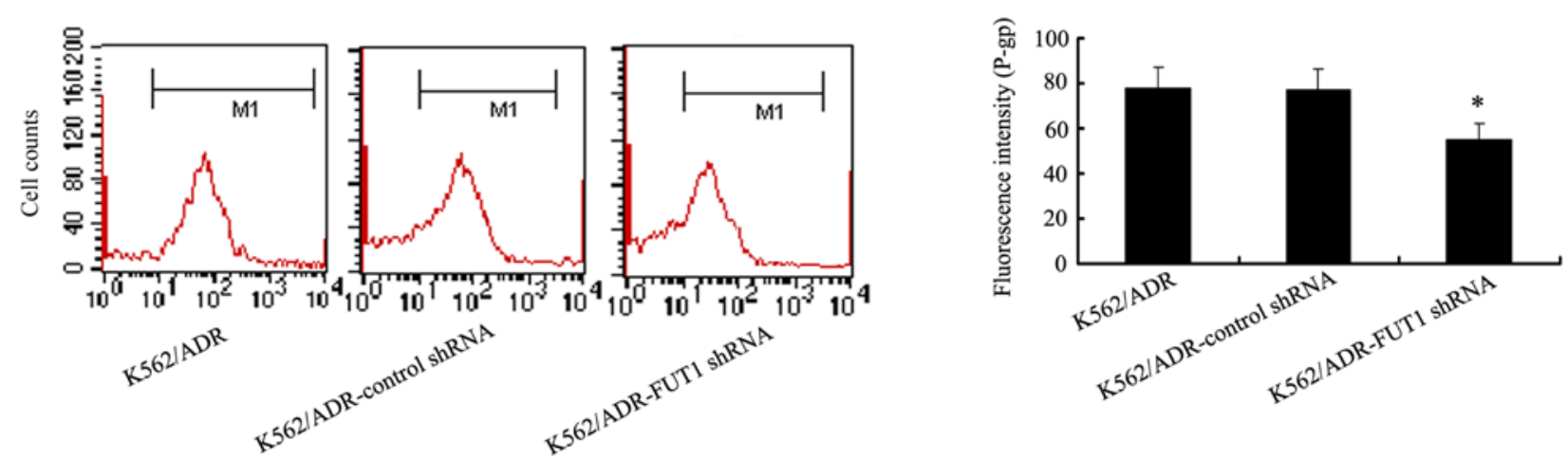

$\mathrm{D}$
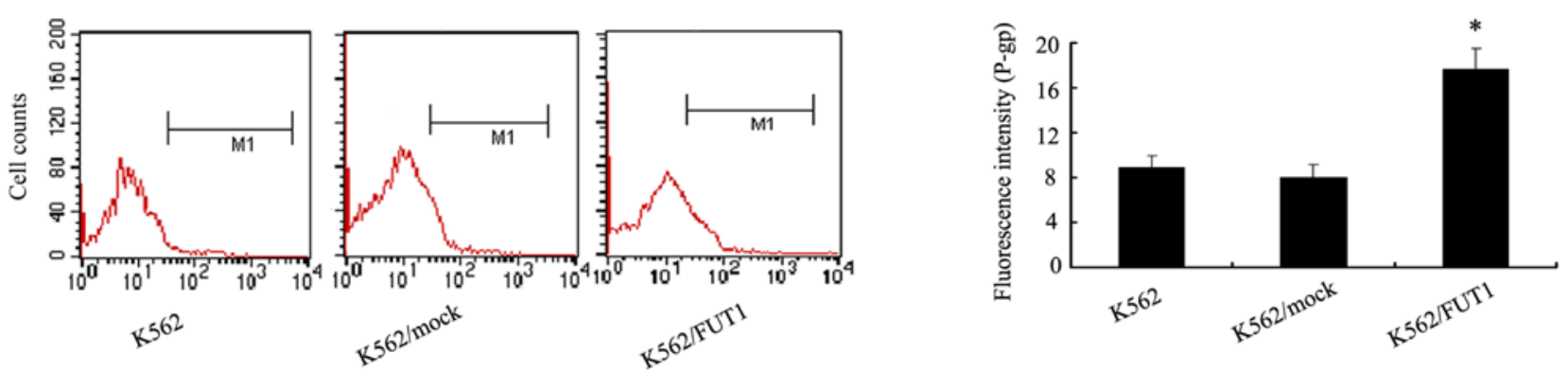

Figure 4. Effect of FUT1-activated EGFR/MAPK signaling pathway on the expression of P-gp. (A) Expression of EGFR/MAPK signaling molecules were repressed at protein levels with FUT1 shRNA transfection in K562/ADR cells. (B) The increased protein levels of EGFR/MAPK signaling molecules were determined by western blot analysis in K562/FUT1 cells. (C) Decreased relative expression of P-gp was examined by flow cytometric analysis in FUT1shRNA-treated K562/ADR cells. (D) Flow cytometric analysis revealed a higher relative expression of P-gp in K562 cells with FUT1 transfection. The data are means \pm SD of 3 independent assays ( $(\mathrm{P}<0.05)$.

After FUT1 shRNA transfection, the ability of adriamycin, paclitaxel and vincristine to inhibit the growth of K562/ADR was evaluated by MTT assay. The results showed that $\mathrm{IC}_{50}$ values were significantly decreased in K562/ADR-FUT1 shRNA group compared to the control, suggesting that cell proliferation was inhibited by therapeutic drug and chemo- sensitivity was remarkably restored when FUT1 gene was suppressed (Fig. 2C).

Nude mice bearing K562/ADR, K562/ADR-control shRNA, and K562/ADR-FUT1 shRNA xenografts were used to determine the treatment efficacy of adriamycin by measuring tumor volumes. Fig. 2D showed that a significant 
A

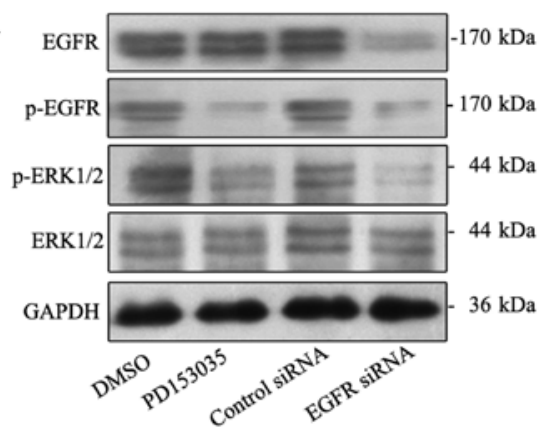

$\mathrm{B}$

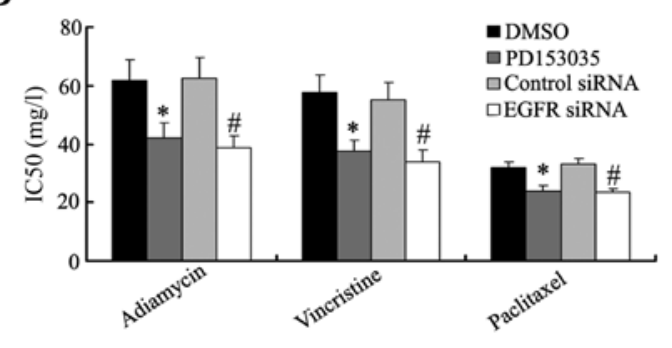

$\mathrm{D}$

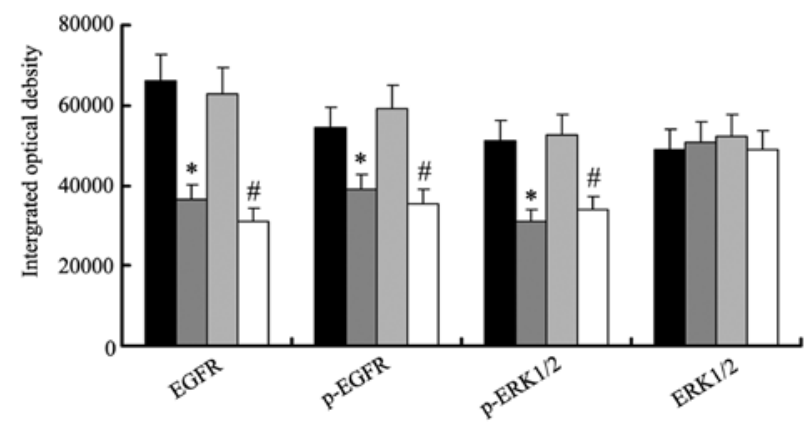

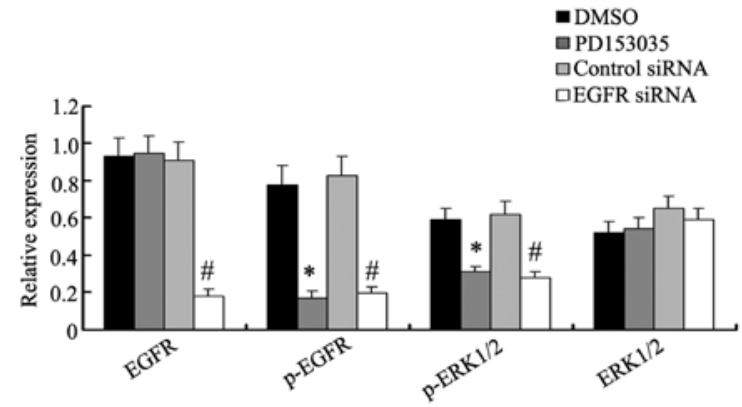

$\mathrm{C}$

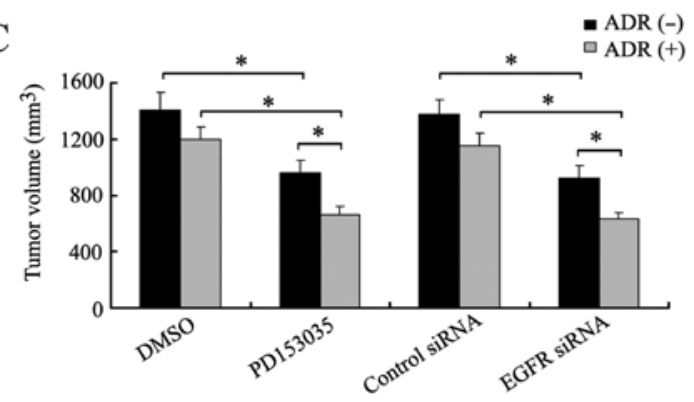

$\mathrm{E}$

Figure 5. Blocking EGFR/MAPK modulates the chemosensitivity of K562/ADR cells both in vitro and in vivo. (A) The K562/ADR cells were pretreated with PD153035 or EGFR siRNA. Expression of EGFR/MAPK signaling molecules were then examined by western blot analysis. (B) PD153035 or EGFR treatment also alleviated chemoresistance of K562/ADR cells, revealed in vitro and in vivo (C). (D) Downregulation of EGFR/MAPK signaling molecules was also shown by IHC staining in xenograft tumors derived from PD153035 or EGFR siRNA treatment cells. (E) Flow cytometric analysis showed that suppression of EGFR/MAPK signaling resulted in reduced level of P-gp. The data are means $\pm \mathrm{SD}$ of 3 independent assays. ${ }^{*} \mathrm{P}<0.05$ vs. DMSO treatment cells; ${ }^{\#} \mathrm{P}<0.05$ vs. control siRNA treatment cells.

reduction of mean tumor volume of K562/ADR-FUT1 shRNA tumor $\left(536 \pm 71 \mathrm{~mm}^{3}\right)$ was observed, as compared with control shRNA group $\left(1121 \pm 125 \mathrm{~mm}^{3}\right)$, and the effect of concomitant application of adriamycin. These data were consistent with the results of in vitro chemosensitivity analysis. IHC staining analysis of the tumor sections revealed that the expression of FUT1 protein was decreased in the mouse group treated with FUT1 shRNA compared to that in the untreated group (Fig. 2E).

Overexpression of FUT1 gene enhances the chemoresistance of K562 cells in vitro and in vivo. After verifying the effect of FUT1 gene downregulation on tumor cell chemosensitivity, we transfected K562 cells with FUT1 expression vector to determine the effect of overexpression of FUT1 on chemoresistance of K562 cells. Notably, the levels of mRNA and protein of FUT1 increased in FUT1 transfectant (Fig. 3A). Fig. 3B also showed that the FUT1 overexpression resulted in an increase of fluorescence intensity (cell surface $\alpha-1,2$ fucose) compared with the K562/mock cells. MTT assays revealed that $\mathrm{IC}_{50}$ values of three drugs were higher in K562/FUT1 group than those in the K562/mock groups, suggesting a positive association between the FUT1 gene expression and chemoresistance of human CML cells (Fig. 3C).

The nude mice inoculated with tumor cells K562, K562/mock and K562/FUT1 were used to measure and compare tumor volumes with or without adriamycin treatment. Fig. 3D showed that in the group of mice bearing K562 tumors, tumor volume was reduced after adriamycin treatment $\left(475 \pm 59 \mathrm{~mm}^{3}\right)$ compared to those without $\left(711 \pm 68 \mathrm{~mm}^{3}\right)$. In the group of mice bearing K562/FUT1 $\left(985 \pm 87 \mathrm{~mm}^{3}\right)$ tumors, tumor volume increased significantly compared to those of the K562/mock group $\left(718 \pm 62 \mathrm{~mm}^{3}\right)$ even after adriamycin treatment $\left(794 \pm 78\right.$ vs. $\left.462 \pm 53 \mathrm{~mm}^{3}\right)$. High expression level of FUT1 protein was illustrated in the tumor cells of K562/FUT1 by IHC staining, as shown in Fig. 3E. Thus, overexpression 
of FUT1 gene in K562 cells led to increasing resistance to adriamycin chemotherapy.

FUT1-induced chemoresistance to CML cell lines is through EGFR/MAPK and P-gp expression. Given the critical role of EGFR/MAPK pathway in controlling cell chemosensitivity, we analyzed whether FUT1 activated the EGFR/MAPK pathway and whether this pathway was involved in FUT1mediated cell chemosensitivity. Fig. 4A showed that the levels of the p-EGFR and p-ERK1/2 were significantly decreased in low K562/ADR-FUT1 shRNA cells. But the total amount of EGFR and ERK1/2 remained unchanged. On the contrary, overexpression of FUT1 in K562 cells significantly enhanced proteins expression of $\mathrm{p}$-EGFR and $\mathrm{p}$-ERK1/2 as illustrated in Fig. 4B.

Furthermore, we investigated whether FUT1 could influence the expression of P-gp. Interestingly, flow cytometric analysis illustrated that low expression level of P-gp was detected in K562/ADR-FUT1 shRNA cells compared to those in control cell groups (Fig. 4C). In contrast, K562 cells expressed high level of P-gp with FUT1 overexpression (Fig. 4D). These data indicated a possible pathogenetic mechanism of MDR development of CML cells.

Blocking EGFR/MAPK modulates the chemosensitivity of K562/ADR cells both in vitro and in vivo. To further investigate what role EGFR/MAPK pathway plays in the signal transduction of FUT1 in K562/ADR cells, the effects of specific inhibitor of EGFR/MAPK or EFGR siRNA to silence EFGR were selected to treat K562/ADR cells. By western blotting, the expression levels of the main signal molecules of EGFR/MAPK pathway apparently decreased in K562/ADR cells treated with PD153035 or EGFR siRNA (Fig. 5A). The inhibition of EGFR/MAPK pathway made the K562/ADR cells susceptible to chemotherapy (Fig. 5B). Accordingly in vivo chemosensitivity analysis revealed that reduced tumor volumes were detected in mouse group bearing K562/ADR tumors with PD153035 $\left(669 \pm 73 \mathrm{~mm}^{3}\right)$ or EGFR siRNA treatment $\left(638 \pm 57 \mathrm{~mm}^{3}\right)$, as compared with DMSO $\left(1206 \pm 98 \mathrm{~mm}^{3}\right)$ or control siRNA group $\left(1156 \pm 95 \mathrm{~mm}^{3}\right)$, and the effect of concomitant application of adriamycin (Fig. 5C). Altered expression levels of the main signal molecules of EGFR/MAPK pathway were also validated in mouse group bearing K562/ADR tumors treated with PD153035 or EGFR siRNA by IHC staining, as shown in Fig. 5D. Moreover, the inhibitor of EGFR/MAPK or silencing EGFR reduced the expression of P-gp (Fig. 5E). The results implicated a role of EGFR/MAPK signaling in regulating P-gp expression and modulating the chemoresistance of K562/ADR cells.

\section{Discussion}

MDR is considered to be a major problem for clinical therapy related to hematological malignancies. Leukemia cells give rise to a series of biological changes during the MDR development. In the present study, we investigated the association between alteration of $\alpha(1,2)$-fucosylation and expression of their related glycogenes as well as the possible mechanism of FUT1 on MDR development in human CML cell lines.
Changes in the surface fucosylation have been detected in colorectal, hepatocellular carcinoma, head and neck and gastric cancer (14-17). The biosynthetic pathway of fucosylated glycans showed great importance of fucosyltransferases. In this study, we found that the expression profiles of $\alpha(1,2)$ fucosyltransferase were remodeled in three pairs of CML cell lines. All MDR cells were characterized by higher levels of FUT1. The expression of FUT2 exhibited no significant difference in three pairs of CML cell lines. In addition, a great number of CML patients were examined and analyzed in the present study, while $>56.4 \%$ of the CML patients were found resistant to the anticancer drugs. FUT1 was expressed at a high level in peripheral blood mononuclear cells of CML/MDR patients. On the basis of the above results, it implied the utilization of FUT1 as a biomarker for clinical diagnosis and prognosis of MDR of CML.

Besides listing the gene profile, we were also interested in the influence of FUT1 on MDR in human CML cells. Here, we targeted FUT1, which was differentially expressed in K562 and K562/ADR cells. The altered level of FUT1 was responsible for changed drug-resistant phenotype of K562 and K562/ADR cells both in vitro and in vivo. FUT1 product also altered remarkably in CML cell lines labeled with FITC-UEA-1 lectin. These results clearly showed that the change in FUT1 expression level had impact in the remodeling of cell surface fucosylated oligosaccharides, which might consequently affect the biological functions of leukemia cells such as MDR.

Several studies have explored a potential association of FUT1 with signaling pathways. The knockdown of FUT1 gene downregulated HER2 signaling via EGFR downregulation and attenuated cell proliferation in the HER2-overexpressing gastric cancer cell line NCI-N87 (18). Knocking down FUT1 expression by short interfering RNA technique dramatically reduced the expression of FUT1 and inhibited human epidermoid carcinoma A431 cells proliferation through decreasing EGFR signaling pathway (19). The EGFR/MAPK signaling pathway also controls the expression and function of many proteins that are necessary for tumor cell multidrug resistance $(20,21)$. In the present study, we evaluated the correlation of the FUT1-mediated EGFR/MAPK signaling pathway with MDR. The resistant cell line K562/ADR, presented higher EGFR/MAPK activity than the sensitive one, which was in accordance with the MDR phenotype. Altered expression of FUT1 markedly modulated the activity of EGFR/MAPK pathway in human CML cell lines. In addition, inhibition of the EGFR/MAPK pathway with EGFR inhibitor PD153035 or EGFR siRNA reversed the chemoresistance of K562/ADR cells. Our results together with the previous findings, explored a possible mechanism of MDR in CML cells that drug resistance might develop and vary via the EGFR/MAPK pathway activated by FUT1 expression. FUT1-modulated CML cell MDR was, at least in part, EGFR/MAPK-dependent.

Increasing evidence indicates that the EGFR/MAPK pathway enhances drug efflux by ATP-binding cassette (ABC) transporters, maintaining MDR of tumor cells $(22,23)$. Activation of the tyrosine kinase pathway by EGF induced MDR by upregulating the $\mathrm{ABC}$ protein expression and enhanced the survival of resistant HCC cells. In contrast, EGFR inhibition restored chemosensitivity (20). Moreover, reports revealed that activation of the EGFR-pathway increased P-gp expression in 
colorectal cancer cells and enhanced $A B C C 1$ gene expression in MCF-7 breast cancer cells $(24,25)$. In addition, it has been well demonstrated that the knockdown of FUT1 expression inhibited human epidermoid carcinoma A431 cell proliferation through decreasing EGFR signaling pathway (19). Therefore, a close association is indicated between the level of FUT1 and the levels of phosphorylated EGFR, as well as P-gp expression in cancer cells. In the present study, we found that the level of P-gp had a positive relationship with the expression of FUT1 and the activity of EGFR/MAPK signaling in K562 and K562/ ADR cell lines. Consequently, the MDR mediated by FUT1 was also involved in EGFR/MAPK pathway activation and P-gp expression.

In conclusion, by analyzing the differential expression pattern of $\alpha(1,2)$-fucosyltransferase in three pairs of CML cell lines and in peripheral blood mononuclear cells of the CML patients, at least in this system, FUT1 regulation elucidated the unusual property of association with CML cell MDR via modulating the EGFR/MAPK signaling pathway and P-gp expression. Together, these data increase our understanding of the factors that contribute to the complex regulation of glycosylation in cancer.

\section{References}

1. Sloma I, Jiang X, Eaves AC and Eaves CJ: Insights into the stem cells of chronic myeloid leukemia. Leukemia 24: 1823-1833, 2010.

2. Hart GW: Glycosylation. Curr Opin Cell Biol 4: 1017-1023, 1992.

3. Dunphy WG, Brands R and Rothman JE: Attachment of terminal $\mathrm{N}$-acetylglucosamine to asparagine-linked oligosaccharides occurs in central cisternae of the Golgi stack. Cell 40: 463-472, 1985.

4. Kelly RJ, Rouquier S, Giorgi D, Lennon GG and Lowe JB: Sequence and expression of a candidate for the human Secretor blood group alpha(1,2)fucosyltransferase gene (FUT2). Homozygosity for an enzyme-inactivating nonsense mutation commonly correlates with the non-secretor phenotype. J Biol Chem 270: 4640-4649, 1995.

5. Larsen RD, Ernst LK, Nair RP and Lowe JB: Molecular cloning, sequence, and expression of a human GDP-L-fucose:beta-Dgalactoside 2-alpha-L-fucosyltransferase cDNA that can form the $\mathrm{H}$ blood group antigen. Proc Natl Acad Sci USA 87: 6674$6678,1990$.

6. Sun J, Thurin J, Cooper HS, Wang P, Mackiewicz M, Steplewski Z and Blaszczyk-Thurin M: Elevated expression of H type GDP-Lfucose:beta-D-galactoside alpha-2-L-fucosyltransferase is associated with human colon adenocarcinoma progression. Proc Natl Acad Sci USA 92: 5724-5728, 1995.

7. Wu CS, Yen CJ, Chou RH, Chen JN, Huang WC, Wu CY and Yu YL: Downregulation of microRNA-15b by hepatitis B virus $\mathrm{X}$ enhances hepatocellular carcinoma proliferation via fucosyltransferase 2-induced Globo H expression. Int J Cancer 134: $1638-1647,2014$.

8. Iwamori M, Tanaka K, Kubushiro K, Lin B, Kiguchi K, Ishiwata I, Tsukazaki K and Nozawa S: Alterations in the glycolipid composition and cellular properties of ovarian carcinoma-derived RMG- 1 cells on transfection of the $\alpha 1,2$-fucosyltransferase gene. Cancer Sci 96: 26-30, 2005.
9. Hao YY, Lin B, Zhao Y, Zhang YH, Li FF, Diao B, Ou YL and Zhang SL: alpha1,2-fucosyltransferase gene transfection influences on biological behavior of ovarian carcinoma-derived RMG-I cells. Fen Zi Xi Bao Sheng Wu Xue Bao 41: 435-442, 2008 (In Chinese).

10. Fischer OM, Hart S and Ullrich A: Dissecting the epidermal growth factor receptor signal transactivation pathway. Methods Mol Biol 327: 85-97, 2006.

11. Iida M, Brand TM, Campbell DA, Starr MM, Luthar N, Traynor AM and Wheeler DL: Targeting AKT with the allosteric AKT inhibitor MK-2206 in non-small cell lung cancer cells with acquired resistance to cetuximab. Cancer Biol Ther 14: 481-491, 2013.

12. Fan P, Wang J, Santen RJ and Yue W: Long-term treatment with tamoxifen facilitates translocation of estrogen receptor alpha out of the nucleus and enhances its interaction with EGFR in MCF-7 breast cancer cells. Cancer Res 67: 1352-1360, 2007.

13. Kassouf W, Dinney CP, Brown G, McConkey DJ, Diehl AJ, Bar-Eli $\mathrm{M}$ and Adam L: Uncoupling between epidermal growth factor receptor and downstream signals defines resistance to the antiproliferative effect of Gefitinib in bladder cancer cells. Cancer Res 65: 10524-10535, 2005.

14. Lattová E, Tomanek B, Bartusik D and Perreault H: N-glycomic changes in human breast carcinoma MCF-7 and T-lymphoblastoid cells after treatment with herceptin and herceptin/Lipoplex. J Proteome Res 9: 1533-1540, 2010.

15. Shu H, Zhang S, Kang X, Li S, Qin X, Sun C, Lu H and Liu Y: Protein expression and fucosylated glycans of the serum haptoglobin- $\{$ beta\} subunit in hepatitis B virus-based liver diseases. Acta Biochim Biophys Sin (Shanghai) 43: 528-534, 2011.

16. Mejías-Luque R, López-Ferrer A, Garrido M, Fabra A and de Bolós C: Changes in the invasive and metastatic capacities of HT-29/M3 cells induced by the expression of fucosyltransferase 1. Cancer Sci 98: 1000-1005, 2007.

17. Vasseur JA, Goetz JA, Alley WR Jr and Novotny MV: Smoking and lung cancer-induced changes in N-glycosylation of blood serum proteins. Glycobiology 22: 1684-1708, 2012.

18. Kawai S, Kato S, Imai H, Okada Y and Ishioka C: Suppression of FUT1 attenuates cell proliferation in the HER2-overexpressing cancer cell line NCI-N87. Oncol Rep 29: 13-20, 2013.

19. Zhang Z, Sun P, Liu J, Fu L, Yan J, Liu Y, Yu L, Wang X and Yan Q: Suppression of FUT1/FUT4 expression by siRNA inhibits tumor growth. Biochim Biophys Acta 1783: 287-296, 2008.

20. Hoffmann K, Xiao Z, Franz C, Mohr E, Serba S, Büchler MW and Schemmer P: Involvement of the epidermal growth factor receptor in the modulation of multidrug resistance in human hepatocellular carcinoma cells in vitro. Cancer Cell Int 11: 40, 2011.

21. Wang X, Martindale JL and Holbrook NJ: Requirement for ERK activation in cisplatin-induced apoptosis. J Biol Chem 275: 39435-39443, 2000

22. Barancík M, Bohácová V, Kvackajová J, Hudecová S, Krizanová O and Breier A: SB203580, a specific inhibitor of p38-MAPK pathway, is a new reversal agent of P-glycoproteinmediated multidrug resistance. Eur J Pharm Sci 14: 29-36, 2001.

23. Yang JM, Vassil AD and Hait WN: Activation of phospholipase $\mathrm{C}$ induces the expression of the multidrug resistance (MDR1) gene through the Raf-MAPK pathway. Mol Pharmacol 60: 674-680, 2001

24. Katayama K, Yoshioka S, Tsukahara S, Mitsuhashi J and Sugimoto Y: Inhibition of the mitogen-activated protein kinase pathway results in the down-regulation of P-glycoprotein. Mol Cancer Ther 6: 2092-2102, 2007.

25. Garcia R, Franklin RA and McCubrey JA: EGF induces cell motility and multi-drug resistance gene expression in breast cancer cells. Cell Cycle 5: 2820-2826, 2006. 